\title{
Medicinal potential of extracts from the chanterelle mushroom, Cantharellus cibarius (Review) and prospects for studying its strains from differs plant communities of ultra- continental regions of the Asia
}

Vyacheslav Vlasenko ${ }^{1 *}$, Dejidmaa Turmunkh ${ }^{2}$,Enkhtuya Ochirbat $^{3}$, Dondov Budsuren ${ }^{2}$, Kherlenchimeg Nyamsuren ${ }^{3}$, Javkhlan Samiya ${ }^{3}$, Burenbaatar Ganbaatar ${ }^{3}$, and Anastasia Vlasenko $^{1}$

${ }^{1}$ Central Siberian Botanical Garden SB RAS, 630090 Zolotodolinskaya st., 101, Novosibirsk, Russia. ${ }^{2}$ Plant Protection Research Institute MULS, 17024, Khoroo 11, Khan-Uul district, Ulaanbaatar, ${ }^{3}$ Institute of General and Experimental Biology MAS, Peace Avenue-54b, Bayanzurkh District, Ulaanbaatar-13330, Mongolia

\begin{abstract}
The article reviews medicinal properties of Cantharellus cibarius. Fruiting bodies of this species are a promising source of extracts with vitamins, carbohydrates, proteins, polysaccharides, sterols, phenolic acids, which various biological properties.
\end{abstract}

\section{Introduction}

Mushrooms are becoming more and more important in our diet for their nutritional characteristic. Basidiomycetes contain a wide range of various drugs such as polysaccharides, organic acids, lipids, steroids, tetracyclic triterpenes, which are of interest for medical applications [1,2].

One of the most frequently harvested mushrooms in forests of ultra-continental regions of the Asia is Yellow chanterelle, C. cibarius (fig. 1) is an edible mushroom with medicinal value.

Chanterelle is a beneficial nutrient for human health with high levels of vitamins B and $\mathrm{C}$, contains a great number of carbohydrates and proteins and a low amount of fat, phenolic compounds and organic acids [3].

This species is appreciated because of the aroma and taste of its fruiting bodies. C. cibarius is one of the most important wild edible mushrooms, therefore, the assessment of its biological properties is of great interest to know its potential as an alternative treatment to chemopreventive strategies when it is consumed as part of a diet.

\section{Materials and methods}

\footnotetext{
*Corresponding author: vlasenkomyces@mail.ru
} 
An up-to-date review based on published data presents the research of main medicinal properties of extracts from fruiting bodies and mycelium of C. cibarius. Information on biological activity of C. cibarius is given in chronological order.

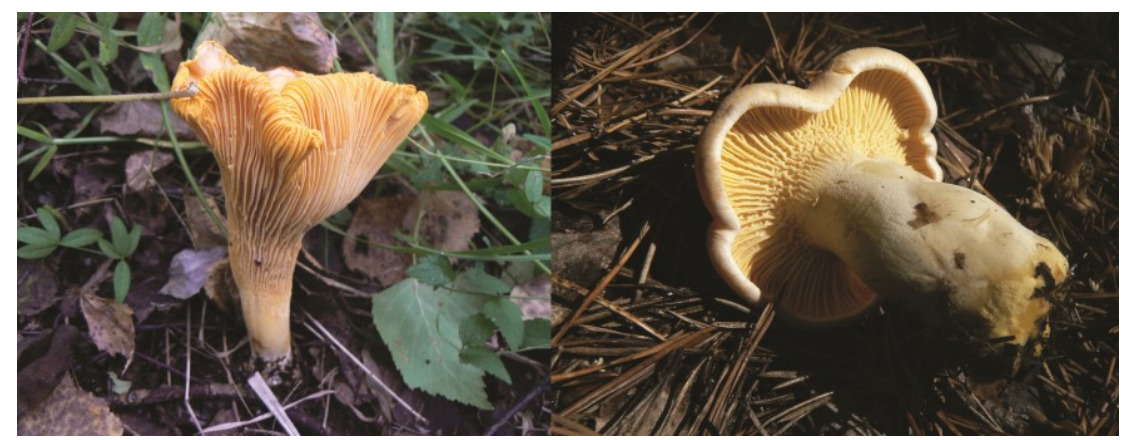

Fig. 1. Fruiting bodies of Cantharellus cibarius in nature.

\section{Results and Discussion}

Cantharellus cibarius has been reported to display a wide variety of biological properties, including antimicrobial, cytotoxicity, antioxidant, antihypoxic, antihyperglycemic, wound healing, anti-inflammatory, iron-chelation activity.

Ethyl acetate, acetone, chloroform and ethanol extracts of $C$. cibarius were tested for antimicrobial activity. Antimicrobial activity against some Gram (+) and Gram (-) bacteria, yeasts, filamentous fungi and actinomycetes was revealed [4].

Many natural foods (including antioxidants) with anti-cancer and anti-inflammatory activity inhibit NF- $k$ B. The inhibitory activities of the extracts of $C$. cibarius and isolated compounds were investigated in an enzyme-based ELISA NF- $k \mathrm{~B}$ assay. Of the tested compounds, ergosterol, ergosterol peroxide and cerevisterol were noted to have the most potent inhibition of NF- $\kappa \mathrm{B}$ activation [5].

Antioxidant activity and bioactive compounds from $C$. cibarius was studied. High phenol and flavonoid content with promising antioxidant properties was shown. Methanolic extracts of wild mushroom $C$. cibarius showed significant antimicrobial activity against $B$. subtilis, S. aureus, E. coli, P. aeruginosa and Candida albicans [6].

Anti-inflammatory properties related to the activity of phenolic compounds originating from edible mushroom C. cibarius were tested in vitro by using LPS-activated RAW 264.7 macrophages. Experiments demonstrated the influence of extracts on the expression of inflammation markers, such as IL-1 $\beta$ and IL-6, and the production of nitric oxide (NO) [7].

Fatty acids contained in mushrooms are capable of supporting anti-inflammatory processes in humans owing to their high content of unsaturated fatty acids. Extracts of $C$. cibarius demonstrated the presence of fatty acids exhibiting antagonist activity toward receptors activated by peroxisome proliferator-activated receptor $\gamma$ (PPAR $\gamma$ ). PPAR $\gamma$ agonists inhibit the development of insulin resistance, thus being an efficient anti-diabetic element, and also demonstrate a therapeutic effect in the case of diseases associated with inflammation and certain tumors [8].

Phenolic acids possess valuable biologically properties: anti-inflammatory, antioxidant, anticarcinogenic and others. One of the most active antioxidants found in mushrooms, caffeic acid, was found in C. cibarius. It exhibits both anti-inflammatory and antioxidative activities $[9,10]$.

Water extracts of $C$. cibarius were studied. Comet assay was used to evaluate the antigenotoxic properties of several concentrations of aqueous extracts. As a test system 
human mononuclear cells exposed to methyl methanesulphonate (MMS) in vitro were used. Extracts showed a decrease in genotoxic properties of MMS [11].

C. cibarius extract is valuable source of antihypoxic activity. Extract showed protective effect against hypoxia in some models. In particular, it produced a significant and dosedependent antihypoxic effect in the hemic and circulatory hypoxia models. Pharmacological effects attributable to the presence of polyphenols in the extract [12].

Methanolic and ethyl acetate extracts of $C$. cibarius exhibit potency to chelate excessive iron in mice. Iron-overloaded animals treated with the extract, showed a dramatic decrease in plasma iron content when compared with the control group. The highest activity was observed in the methanolic extract [13].

The inhibitory effect of pure liquid cultures of $C$. cibarius was tested in vitro against 16 pathogenic Gram-positive and Gram- negative bacterial strains, and 4 strains of oval fungi of the genus Candida. Significant inhibitory effect of the culture fluids of C. cibarius against all tested microbial species was determined. MIC50 of the cultures were similar and lower than those of control broad-spectrum antibiotics thiamphenicol and gentamicin. Liquid cultures of $C$. cibarius can be used to produce effective antimicrobial drugs with broad spectrum activity against bacteria and fungi [14].

Various fractions of the methanolic extract including $n$-hexane, chloroform, ethyl acetate, n-butanol, and water, were evaluated for antioxidant activity. The n-hexane fraction had the highest amounts of flavonoids contents, nitric oxide scavenging activity, and total phenols. However, it was the ethyl acetate fraction that showed the highest DPPH scavenging activity, although the aqueous fraction displayed higher reducing power [15].

The antioxidant, antihyperglycemic and antimicrobial effects of both ethanolic and hydromethanolic extracts of the fruiting bodies of $C$. cibarius were evaluated. Ethanolic extract showed high ferrous ion chelating, 15-lipoxygenase and aglucosidase inhibitory activities. Ethanolic extracts were more active against Staphylococcus aureus ATCC 25923 than the hydromethanolic ones [16].

C. cibarius was analyzed for their fatty acid compositions and antioxidant capacities. Very high metal chelating activity was found. Mushrooms have a potential to be a natural antioxidant in food industries as antioxidant agent [17].

The experimental data obtained in rats show that the extract of $C$. cibarius showed a significant wound healing and anti-inflammatory effect, which can become a scientific justification for the medicinal use of the golden chanterelle mushroom in the treatment of wounds [18].

Activities of cyclohexane, dichloromethane, methanol and aqueous extracts of $C$. cibarius were tested. Broth microdilution assay was performed against 10 bacterial, with emphasis on Helicobacter pylori. Methanol extract was the most active against $H$. pylori strains with minimal inhibitory concentration values between 4 and $32 \mu \mathrm{g} / \mathrm{mL}$. All extracts were active against antibiotic resistant $H$. pylori. Chemical analysis has shown the presence of linoleic, $c i s$-vaccenic, and oleic acids, sterols, $\beta$-glucans, and polyphenolic compounds [19].

The extracts of C. cibarius were used for the evaluation of their effects on cell viability of four cancer cell lines (A172 glioblastoma, U87 glioblastoma, CH157-MN meningioma and PAN-C1 pancreatic) and one NIH3T3 fibroblast cell line. Treatments with high concentrations of $C$. cibarius extracts resulted in significantly reduced number of viable cancer cells for all cell lines tested [20].

\section{Conclusions}

Medicinal properties of $C$. cibarius is of great interest to know its potential as an alternative treatment to chemopreventive strategies when it is consumed as part of a diet. The data on 
the antiviral activity of chanterelles are absent. Therefore, we plan to study the antiviral activity of extracts from fruiting bodies of $C$. cibarius from ultra-continental regions of the Asia (Russia and Mongolia), in which this species is a valuable food resource.

The reported research was funded by Russian Foundation for Basic Research, grant № 19-54-44002 Mong_T.

\section{References}

1. T.V. Teplyakova, N.V. Psurtseva, T.A. Kosogova, N.A. Mazurkova, V.A. Khanin, V.A. Vlasenko, Int. J. Med. Mushr., 14(1) (2012)

2. V.A. Vlasenko, A.V. Vlasenko, BIO Web Conf., IV (2018)

3. B. Muszyńska, K. Sułkowska-Ziaja, H. Ekiert, Acta Scient. Polon. Hortor. Cult., 12 (2013)

4. B. Dulger, A. Gonuz, F. Gucin, Pakistan J. Biol. Sciences, 7(9) (2004)

5. J.A. Kim, D. Tay, E.C. Blanco, Phytotherapy research, 22(8) (2008)

6. C. Ramesh, M.G. Pattar, 2(2) (2010)

7. Palacios, M. Lozano, C. Moro, M.D’Arrigo, M.A. Rostagno, J.A. Martinez, A. Villares, Food Chemistry, 128 (2011)

8. S.S. Hong, J.H. Lee, W. Jeong, N. Kim, H.Z. Jin, B.Y. Hwang, D. Lee, Bioorg. Med. Chem. Lett, 22 (2012)

9. C. Mendez-Espinoza, E. Garcia-Nieto, A.M. Esquivel, M.M. Gonzalez, E.V. Bautista, C.C. Ezquerro, L.J. Santacruz, Int. J. Med. Mushrooms, 15(3) (2013)

10. F.S. Reis, A. Martins, L. Barros, I.C.F.R. Ferreira, Food Chem. Toxicol., 50 (2012)

11. C. Mendez-Espinoza, E. Garcia-Nieto, A.M. Esquivel, M.M. Gonzalez, E.V. Bautista, C.C. Ezquerro, L.J. Santacruz, Int. J. Med. Mushrooms, 15(3) (2013)

12. M. Khalili, M.A. Ebrahimzadeh, F. Omrani, M. Karami, 16(4) (2014)

13. M. Khalili, M.A. Ebrahimzadeh, M. Kosaryan, A. Abbasid, M. Azadbakhta, RSC Advances, 5 (2015)

14. T.P. Popova, Int. J. Curr. Microbiol. App. Sci, 4(3) (2015)

15. M.A. Ebrahimzadeh, Y. Safdari, M. Khalili, Int. J. Med. Mushrooms, 17 (2015)

16. D.E. Zavastin, A. Bujor, C. Tuchiluş, C.G. Mircea, S.P. Gherman, A.C. Aprotosoaie, A. Miron, J. Plant Develop., 23 (2016)

17. Türkekul, F. Çetin, M. Elmastaş, J. Appl. Biol. Chem., 60(1) (2017)

18. D. Nasiry, A.R. Khalatbary, M.A. Ebrahimzadeh, Int. J. Med. Mushrooms, 19(10) (2017)

19. M. Kolundžić, T. Stanojković, J. Radović, A. Tačić, M. Dodevska, M. Milenković, F. Sisto, C. Masia, G. Farronato, V. Nikolić, T. Kundaković, J. Med. Food., 20(8) (2017)

20. K.L. Chin, S. Khanal, G.R. Hankins, V. Perla, Y. Qi., Act. Scient. Agriculture, 2(5) (2018) 